\title{
Adventitious root formation in rice requires OsGNOM1 and is mediated by the OsPINs family
}

\author{
Shiping Liu ${ }^{1,2}$, Jirong Wang ${ }^{1}$, Lu Wang ${ }^{1}$, Xiaofei Wang ${ }^{1}$, Yanhong Xue ${ }^{1}$, Ping Wu ${ }^{1}$, Huixia Shou ${ }^{1}$ \\ ${ }^{1}$ State Key Laboratory of Plant Physiology and Biochemistry, College of Life Science, Zhejiang University, Hangzhou 310058, \\ China; ${ }^{2}$ College of Chemistry and Life Science, China Three Gorges University, Yichang 443002, China
}

The fibrous root system in cereals comprises primarily adventitious roots (ARs), which play important roles in nutrient and water uptake. Current knowledge regarding the molecular mechanism underlying AR development is still limited. We report here the isolation of four rice (Oryza sativa L.) mutants, from different genetic backgrounds, all of which were defective in AR formation. These mutants exhibited reduced numbers of lateral roots (LRs) and partial loss of gravitropism. The mutants also displayed enhanced sensitivity to $\boldsymbol{N}$-1-naphthylphthalamic acid, an inhibitor of polar auxin transport (PAT), indicating that the mutations affected auxin transport. Positional cloning using one of the four mutants revealed that it was caused by loss-of-function of a guanine nucleotide exchange factor for ADPribosylation factor (OsGNOM1). RT-PCR and analysis of promoter::GUS transgenic plants showed that OsGNOM1 is expressed in AR primordia, vascular tissues, LRs, root tips, leaves, anthers and lemma veins, with a distribution pattern similar to that of auxin. In addition, the expressions of $O s P I N 2, O s P I N 5 b$ and $O s P I N 9$ were altered in the mutants. Taken together, these findings indicate that OsGNOM1 affects the formation of ARs through regulating PAT.

Keywords: Oryza sativa L., adventitious root, OsGNOM1, polar auxin transport

Cell Research (2009) 19:1110-1119. doi: 10.1038/cr.2009.70; published online 23 June 2009

\section{Introduction}

In contrast to the tap root system of Arabidopsis thaliana, monocot cereals consist almost entirely of a complex fibrous root system, comprising a mass of adventitious roots (ARs) that develop from the stem base postembryonically. Rice (Oryza sativa L.) is a model cereal crop with seminal roots that die during the growing period [1]. Thus, lateral roots (LRs) and AR are the key determinants of nutrient and water use efficiency in rice.

A number of Arabidopsis mutants with defects in root development have been characterized [2, 3]. These research efforts have comprehensively documented the molecular control and hormonal regulation of root initiation and development in dicotyledonous plants [3-5]. In recent years, an increasing number of cereal mutants

Correspondence: Ping $\mathrm{Wu}^{\mathrm{a}}$, Huixia Shou ${ }^{\mathrm{b}}$

${ }^{\mathrm{a}}$ Fax: +86 571 88206146;

E-mail: clspwu@zju.edu.cn

${ }^{b} E$-mail: huixia@zju.edu.cn

Received 15 December 2008; revised 9 March 2009; accepted 24 March 2009; published online 23 June 2009 with impaired root formation have been discovered [69]. However, due to the complex structure and intricate regulation of their root systems, the mechanism of AR development is still far from clear.

Phytohormone is required for the establishment of appropriate root architecture. It affects the number and length of LR and AR [2]. Many genes required in root development have connections with the auxin-signaling pathway $[4,10]$, including the Aux/IAA family [11], the auxin response factor family [12] and certain hormonerelated transporters [13]. An LOB domain-containing transcription factor controlled by auxin responsive factor was known to play a critical role in the formation of AR primordia in rice and maize (Zea mays L.) [8-9, 14].

GNOM is a large guanine nucleotide exchange factor (GEF) for ADP-ribosylation factor (ARF), a small GTPase. GNOM affects polar auxin transport (PAT) and development in Arabidopsis [15-18]. Over 20 allelic mutants of gnom have been identified [19-21]. Most of these mutants exhibited severe defects in both early embryonic structure and subsequent post-embryonic development such as LR formation and gravitropism, resulting in lethality $[19,22,23]$. GNOM is found in 
endosomal compartments and catalyzes guanine nucleotide exchange through its SEC7 domain, which is highly conserved among animals, plants and fungi [21, 24-26]. Except for GL1 in Arabidopsis, most large ARF-GEFs in plants (including GNOM) are predicted to be sensitive to the fungal toxin brefeldin A (BFA), a specific inhibitor of membrane protein trafficking $[27,28]$. BFA treatment can change the cellular localization of GNOM, yielding a mutant phenotype similar to that of gnom mutants [18]. BFA also regulates vesicle formation by activating ARF GTPases on specific membranes [18, 27, 29]. In gnom mutants, the auxin efflux carrier component PIN1 (PINFORMED 1) is mislocated, suggesting that GNOM regulates the cellular distribution and trafficking of PIN1 in Arabidopsis [30-32].

In this study, we isolated four lethal rice mutants from mutant libraries generated from japonica variety Zhonghua11 and from indica variety Kasalath. These mutants have reduced numbers of LR and lack AR. The phenotypes are similar to those of wild-type (WT) plants treated with $N$-1-naphthylphthalamic acid (NPA), an inhibitor of PAT. Using a positional cloning strategy, we identified that the OsGNOM1 gene is responsible for the mutations. We also found that the transcript levels of OsPIN2, OsPIN5b and OSPIN9 were altered in mutants in the basal portion of the shoots where the AR primordia are initiated, suggesting that OsGNOM1 is required to maintain the normal distribution of auxin during the initiation of AR.

\section{Results}

Isolation and characterization of gnom1 rice mutants

Four rice mutants, here designated as gnom1-1 to gnom1-4 (see later for the cloning of OsGNOM1), were isolated from mutant libraries generated from japonica variety Zhonghua11 (gnom 1-1) and indica variety Kasalath (gnom 1-2, gnom 1-3 and gnom 1-4), as described in Table 1. These mutants lacked AR during the early stages of growth and exhibited a reduced number of LR and partial agravitropism (Figure $1 \mathrm{~A}$ to $1 \mathrm{D}$, and Supplementary information, Table S1). A small number of AR emerged at 6 weeks after germination (Figure
1E), whereas no mutants survived longer than 2 months. When all roots from the base of the stem were surgically removed, the WT seedlings initiated new roots in 7 days but the mutants did not (Figure 1F), indicating that these mutants had lost the ability to regenerate new roots.

Longitudinal and cross-sections obtained from the basal portion of the stem of 5-day-old seedlings showed that AR primordia formation was impaired in the gnom 1 mutants (Figure 1G to $1 \mathrm{~J}$ ). Methylene blue staining [33] of seminal roots showed that the number of LR primordia was also significantly reduced (Figure $1 \mathrm{~K}$ and $1 \mathrm{~L}$ ).

\section{Genetic analysis}

The OsGNOM1 locus was cloned using a map-based cloning strategy. The $\mathrm{F}_{2}$ segregation population derived from a cross between a heterozygous gnom 1-1 and the indica cultivar Kasalath was used for mapping. AR development was normal in the $\mathrm{F}_{1}$ generation, indicating that a recessive mutation controlled the phenotype. OsGNOMI was mapped to the long arm of chromosome 3 between the single sequence repeat (SSR) marker RM15621 and a sequence-tagged site (STS) marker STS18 using $120 \mathrm{mu}$ tants (Figure 2A). To finely map this locus, 962 mutant seedlings were further analyzed using three newly developed STS markers: STS2, STS14 and STS19. Sequencing the genomic region between STS2 and STS14 in WT and mutant plants revealed that there was a guanine deletion in the third exon of the OSGNOM1 gene (accession number: EAY91294.1, Figure 2B); the deletion results in a frame shift. Subsequent sequencing of OsGNOM1 in three other mutants (gnom1-2, gnom 1-3 and gnom14) confirmed mutations in the OsGNOM1 gene. gnom 1-2 has a C/A substitution at position 4447 , resulting in premature termination of protein synthesis (Figure $2 \mathrm{~B}$ and Table 1). gnom 1-3 and gnom 1-4 have GA and TG deletions at positions 5799 and 3 107, respectively (Figure 2B and Table 1). These deletions result in frameshift and the formation of non-functional Os GNOM1 proteins.

Alignment of OsGNOM1 with GNOM in Arabidopsis revealed the presence of three exons encoding six conserved domains (Figure 2B). Both gnom 1-1 and gnom 1-2 involves mutations in the HDS1 (homology downstream of SEC7) domain, while gnom1-3 and gnom 1-4 have a

Table 1 Features of gnom 1 alleles in rice

\begin{tabular}{|c|c|c|c|c|}
\hline Mutant name & gnom1-1 & gnom 1-2 & gnom 1-3 & gnom 1-4 \\
\hline Genetic background & Zhonghua 11, Japonica & Kasalath, Indica & Kasalath, Indica & Kasalath, Indica \\
\hline Mutagen & Tissue culture & EMS & $\gamma$ radiation & $\gamma$ radiation \\
\hline Position & 4328 & 4447 & 5799 & 3107 \\
\hline Domain & HDS1 & HDS1 & HDS3 & HUS \\
\hline
\end{tabular}



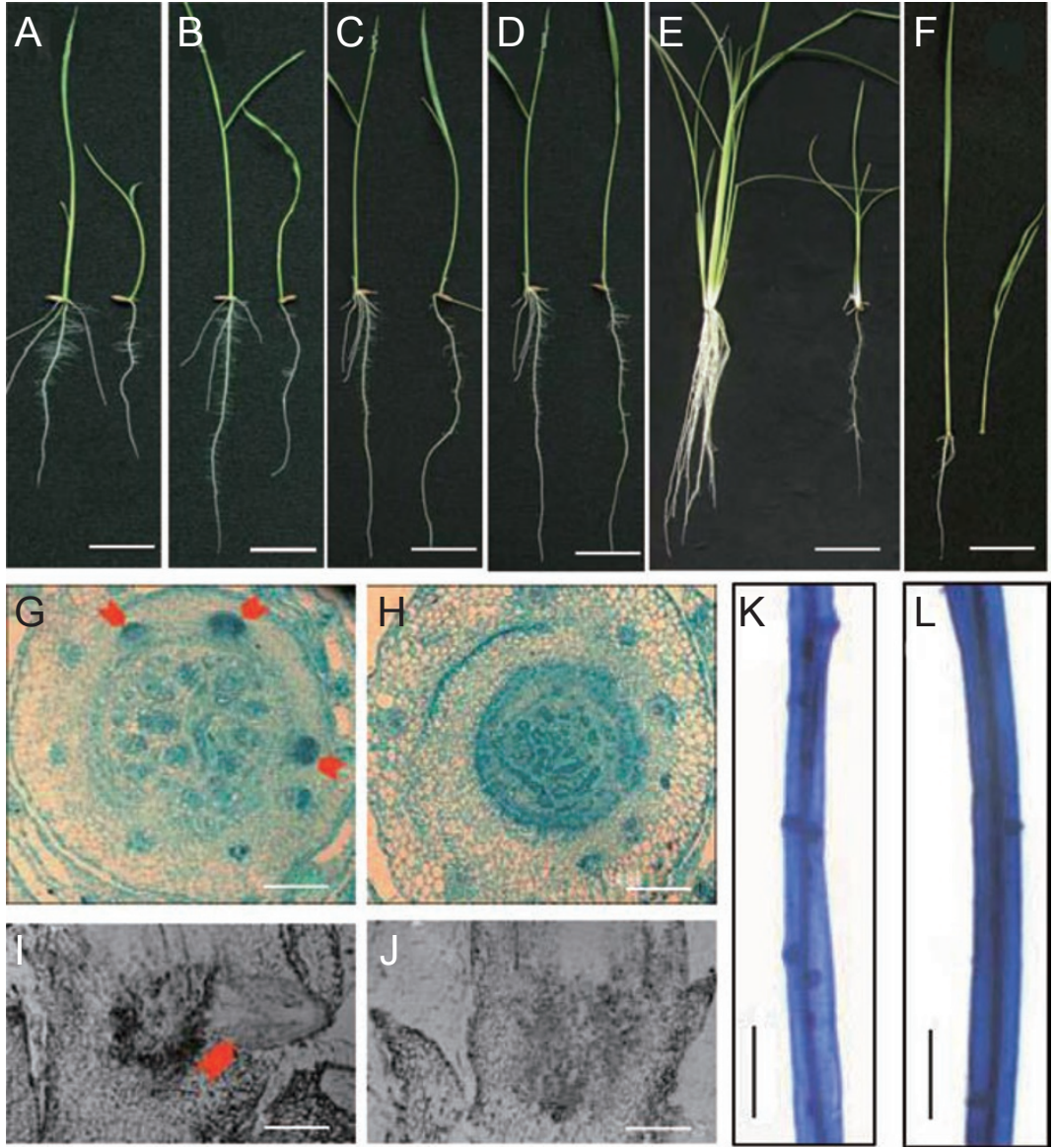

Figure 1 Phenotypes of rice gnom1 mutants. (A-D) Seven-day-old seedlings representing gnom1-1 to gnom1-4. The mutant is on the right and wild type is on the left. Bars $=2 \mathrm{~cm}$. (E) Six-week-old seedlings (left: Zhonghua 11; right: gnom1-1). Bar $=5 \mathrm{~cm}$. (F) WT (left) and gnom1-1 (right) at 1 week after surgically removing roots, Bar $=2 \mathrm{~cm}$. (G-H) Stem-base cross-sections of the WT (G) and gnom1-1 (H); the arrows show the AR primordia. Bars = $100 \mu \mathrm{m}$. (I-J) Stem-base vertical sections of the WT (I) and gnom1-1 (J); the arrows show the AR primordia. Bars $=200 \mu \mathrm{m}$. (K and $\mathrm{L})$ Methylene blue staining of seminal roots of WT (K) and gnom1-1 (L). Bars $=200 \mu \mathrm{m}$.

mutation in the HDS3 and HUS (homology upstream of SEC7) domains (Figure 2B and Table 1), respectively.

To confirm the positional cloning of these mutants, we developed two dCAPS (derived cleavage amplify polymorphism) markers, C1 for gnom 1-1 and C2 for gnom 1-2 (Supplementary information, Figure S1). The heterozygote mutants, homozygote mutants and WT genotypes can be easily distinguished in the segregation population using these dCAPS markers (Figure 2C). Moreover, the mutation of OsGNOM1 in gnom1-3 was also confirmed by $\mathrm{Cel}$ I digestion, a restriction enzyme that digests DNA specifically at mismatch sites (Figure 2D).

Genetic complementation of the gnom 1-1 mutant was carried out by introducing a 4.8-kb WT cDNA fragment of OSGNOM1 into modified pCAMBIA 1300 under control of the $35 \mathrm{~S}$ promoter. After Agrobacterium tumfaciens-mediated transformation, fifteen independent transgenic lines were obtained and confirmed by RT-PCR (Figure 2E). These transgenic plants showed normal development of $A R$, indicating that the mutant phenotype was completely complemented.

\section{Effect of gnom1 mutation on callus initiation}

Although the gnom 1-1 mutant in the japonica background did not affect callus induction, the gnom 1-2 mutation in the indica background significantly reduced the rate of callus induction, as compared to that of the WT counterpart (Figure 3A and 3C). Using the $\mathrm{C} 1$ and C2 dCAPS markers, we were able to distinguish the homozygous mutant calli from the heterozygotes and calculate the frequency of the homozygous mutant genotype among the calli generated from the mixed seeds produced by heterozygotic plants. Results showed that the frequency of homozygous mutant callus in the in- 
A

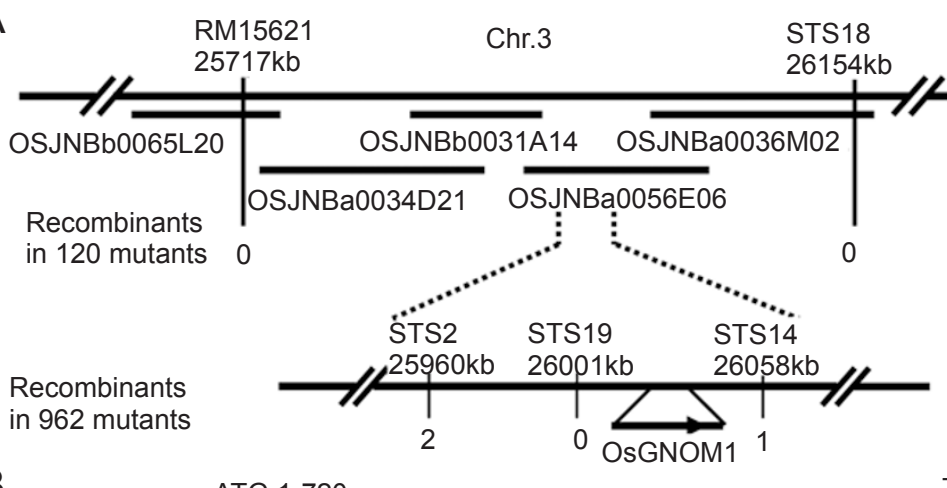

B
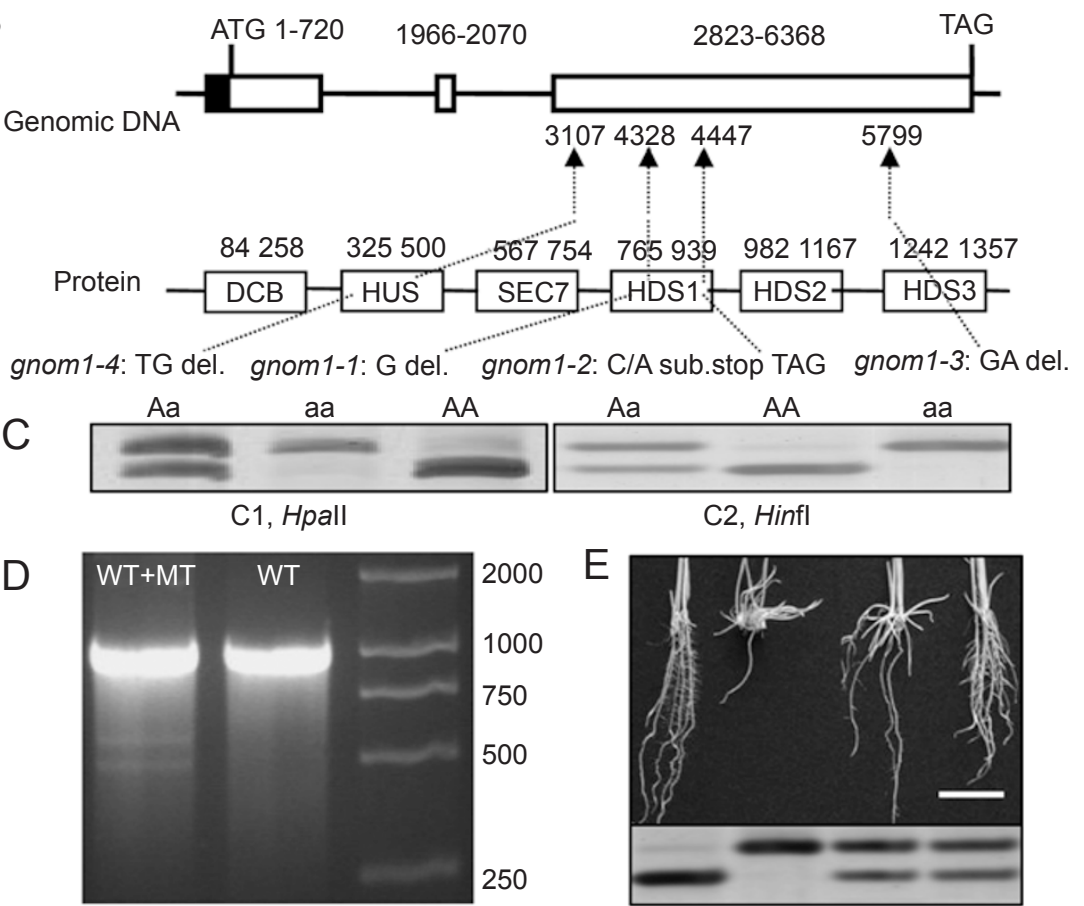

C2, Hinfl

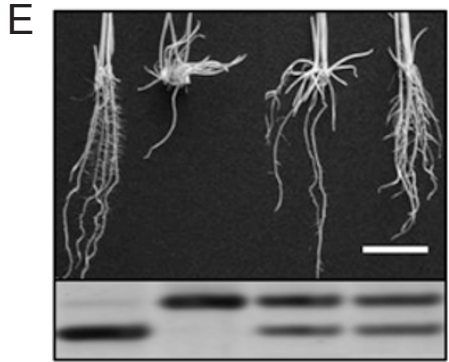

Figure 2 Map-based cloning of the OsGNOM1 gene. (A) Fine mapping of the OsGNOM1 gene between the markers STS19 and STS14 within the BAC clone OSJNBa0056E06. BAC, bacterial artificial chromosome. The number of recombinants is marked corresponding to the molecular markers. (B) Gene and protein structure of the OsGNOM1. The mutation sites of four alleles (gnom1-1 to gnom 1-4) are marked by the arrow. (C) Confirmation of gnom1-1 and gnom1-2 by the dCAPS markers C1 and C2. (D) The OsGNOM1 DNA fragments of gnom1-3 and Kasalath digested with Cel I. (E) Complementation analysis of the gnom1-1 mutant. From left to right in the upper panel: WT plant, gnom1-1 mutant, two lines of gnom1-1 mutant harboring exogenous OsGNOM1. Bar $=2 \mathrm{~cm}$. The bottom panel: C1 PCR products after Hpall digestion using cDNA for OsGNOM1 in PAGE gel.

dica background was significantly lower than the ratio predicted by Mendelian segregation (Figure 3D). Furthermore, in contrast to the normal morphology of indica calli, those homozygous for gnom 1-2 were dispersed and fragile (Figure 3B). However, no difference was observed between WT and gnom 1-1 calli in the japonica genetic background, suggesting that OsGNOM1 may play a different role in the indica and japonica subspecies.

\section{Effect of gnom1 mutation on PAT}

When treated with NPA, the inhibitor of PAT, the WT seedlings exhibited a phenotype similar to that of gnom1. As shown in Figure 4A, neither the NPA-treated WT seedlings nor the gnom 1 seedlings had AR. The aerial parts of both groups of seedlings grew in a curly manner, while the roots in both groups displayed a loss of gravitropism (Figure 4A and 4B). In addition, the mutants were more sensitive to NPA than the WT plants (Figure 4A), indicating that PAT was impaired in the mutants. Howev- 

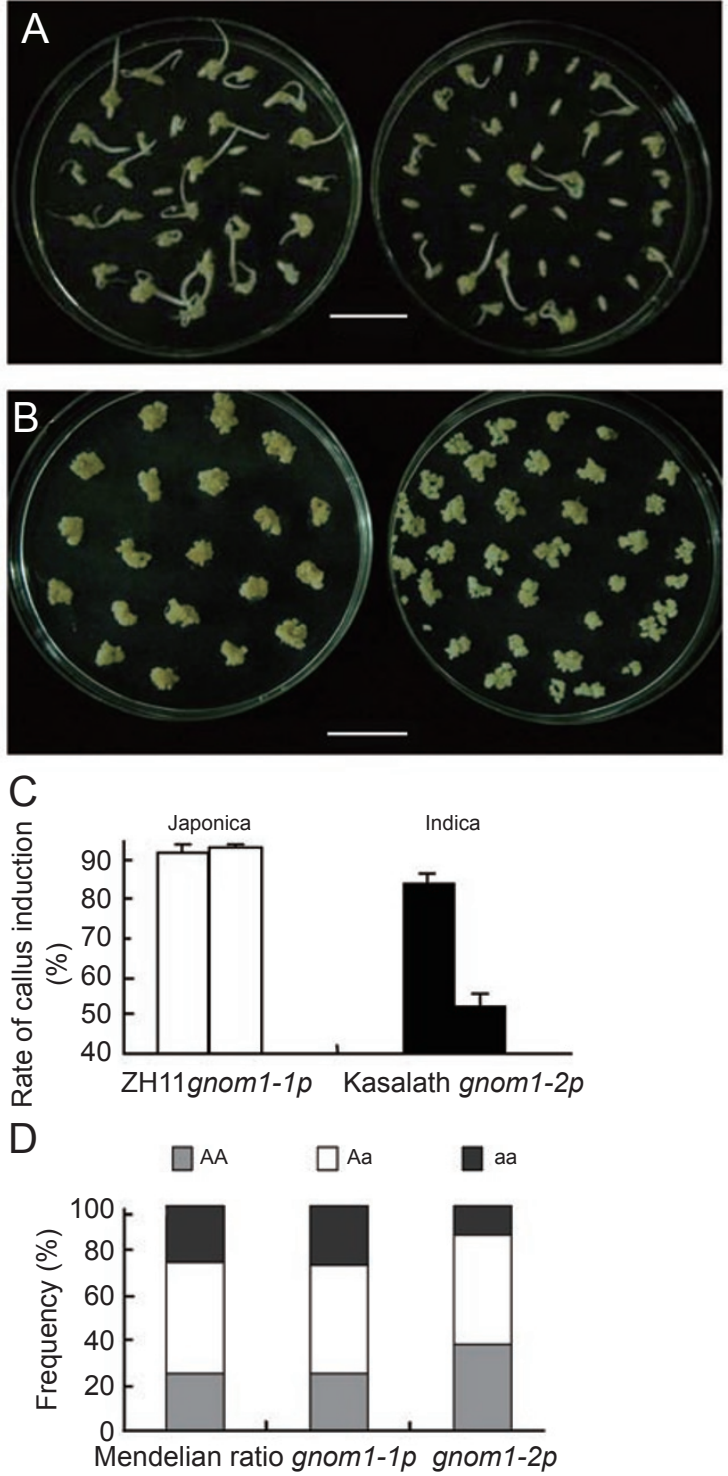

Figure 3 The callus characteristics of indica genotype, Kasalath and gnom1-2. (A) Calli induction from Kasalath WT seeds (left) and from mixed seeds produced by gnom1-2 heterozygotic plants (right). Bars $=2 \mathrm{~cm}$. (B) Morphology of Kasalath calli (left) and homozygous gnom1-2 calli (selected by molecular marker $\mathrm{C} 2$, right). Bars $=2 \mathrm{~cm}$. (C) Rate of callus induction for WT seeds or mixed seeds produced by heterozygous plants, on japonica or indica background. gnom 1-1p and gnom 1-2p denote seeds produced by the gnom1-1 and gnom1-2 heterozygous populations, respectively. (D) The genotype frequency of calli induced from seeds produced by gnom1-1 and gnom1-2 heterozygous populations.

er, the phytohormones $\alpha$-naphthalene acetic acid (NAA) and penetrable 2,4-D did not restore AR development in mutants (Supplementary information, Figure S2).

Suppression of OsPIN1b in WT rice resulted in reduced numbers of AR and increased sensitivity to NPA
[13]. However, semi-quantitative RT-PCR analysis showed that the OsPIN1b transcript level in the basal portion of the shoots where the AR primordia are initiated was almost identical to that of the WT plants (Figure $5 \mathrm{~A})$. To investigate whether OsGNOM1 regulates the ex-

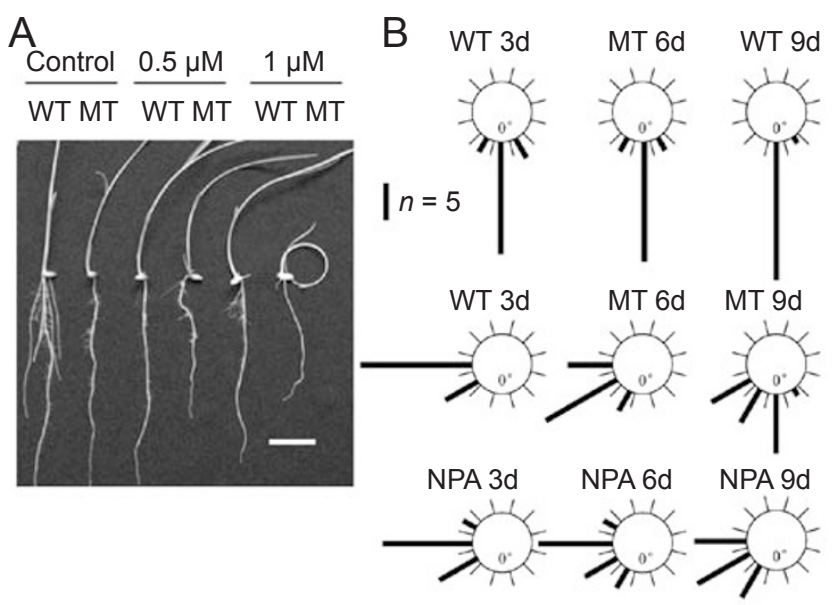

Figure 4 The effects of NPA on WT and gnom1-1. (A) WT and gnom1-1 treated with NPA, and the solvent DMSO as control. Bar $=2 \mathrm{~cm}$. (B) The root gravitropism response of WT, gnom1-1 mutant (MT) and WT treated with $1 \mu \mathrm{M}$ NPA for 3, 6 and 9 days.

A

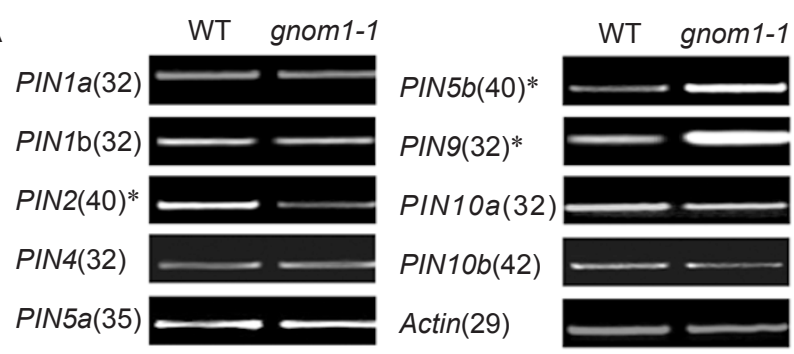

B

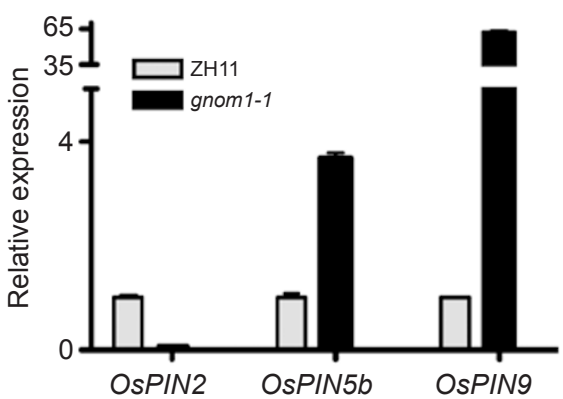

Figure 5 The expression of OsPINs in WT and gnom1-1. (A) The expression of nine OsPIN family members, determined by semi-quantitative RT-PCR analysis of WT and gnom1-1 stem bases; actin served as an internal control. The differentially expressed genes are marked with *, and the PCR cycles are indicated in the brackets. (B) Quantitative RT-PCR of three genes (OsPIN2, OsPIN5b and OsPIN9) in WT and gnom1-1. 
pression of the PIN gene family in rice, RT-PCR analysis was used to compare gnom 1 mutants to WT plants in terms of OsPIN expression in the basal part of the stems. The results showed that three out of the nine tested PIN genes were expressed differentially in the gnom $1 \mathrm{mu}-$ tants and WT plants (Figure 5A). While the expression of OSPIN2 was downregulated in the mutants, OsPIN5b and OSPIN9 were both upregulated (Figure 5A). The differential expression of these genes was further confirmed by quantitative RT-PCR analysis (Figure 5B). These results showed that expression of the PIN gene family was disrupted in the roots of gnom 1-1 mutants, further supporting the idea that PAT is affected in these plants.

\section{OsGNOM1 expression pattern}

Semi-quantitative RT-PCR analysis was performed

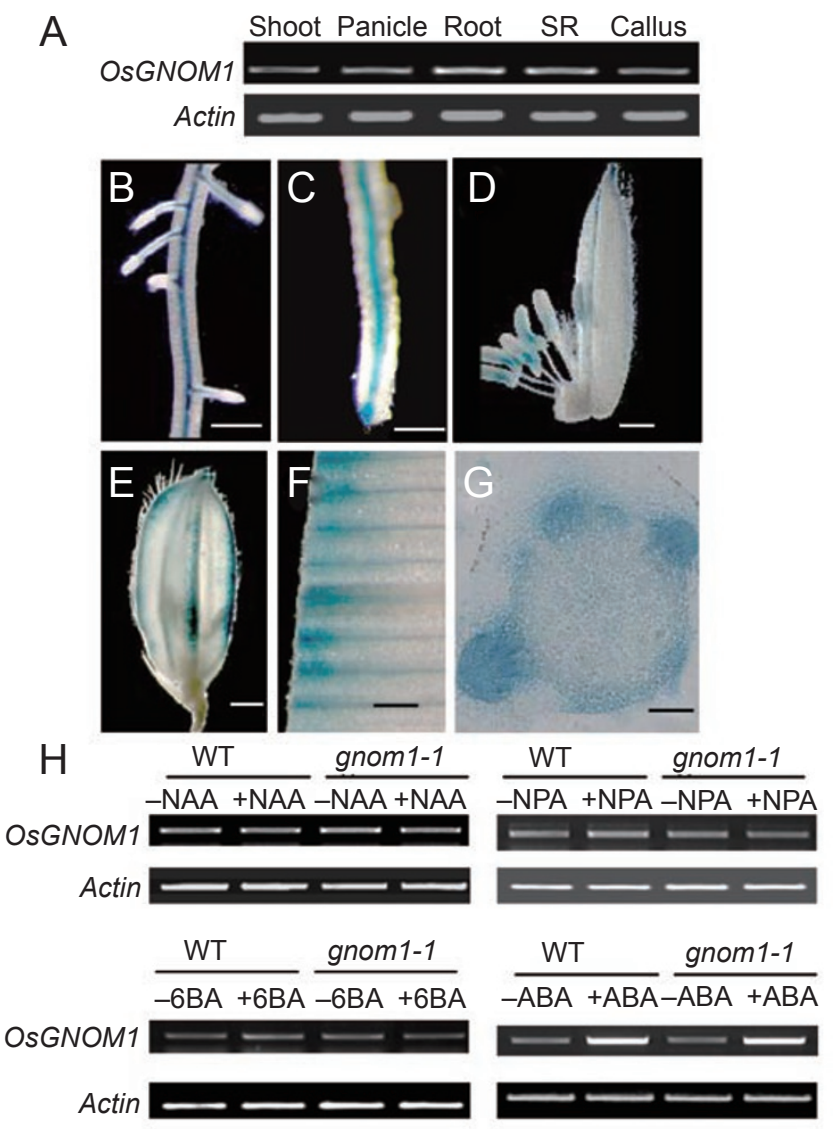

Figure 6 Expression pattern of OsGNOM1. (A) Semi-quantitative RT-PCR of OsGNOM1 in various tissues, and actin as a control. (B-G) OsGNOM1 promoter-driven GUS expression in roots and lateral roots (B), root tips (C), anthers (D) lemma veins $(\mathbf{E})$, leaves $(\mathbf{F})$ and stem-base cross-section $(\mathbf{G})$. Bars in $B, C=200 \mu \mathrm{m}$; in D, E = $500 \mu \mathrm{m}$; in F, G = $100 \mu \mathrm{m}$. (H) Semiquantitative RT-PCR of OsGNOM1 following various hormone treatments.

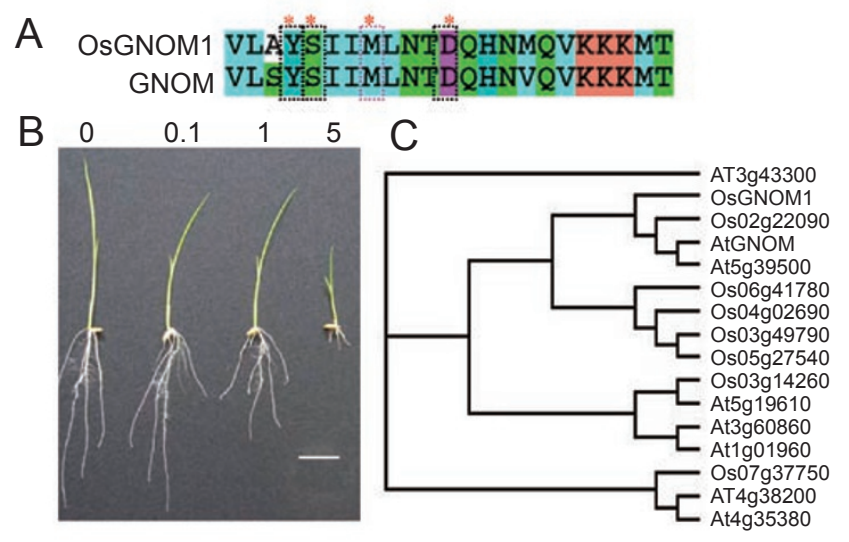

Figure 7 The response of OsGNOM1 to BFA. (A) The alignment of OsGNOM1 and GNOM in SEC7 domain conserved amino acids, which predict sensitivity to BFA. The crucial amino acids are marked by *. (B) The wild-type seedling response to 0 (only solvent DMSO), 0.1, 1 and $5 \mu \mathrm{M}$ BFA. Bar $=2 \mathrm{~cm}$. (C) Dendrogram of the GNOM family proteins in rice and Arabidopsis.

to investigate the expression pattern of $O S G N O M 1$ in various tissues. Results showed that $O s G N O M 1$ was transcribed in all the tissues tested (Figure 6A). Expression of the gene was slightly higher in root and basal internodes than in shoot, panicle or callus, implying that GNOM1 plays important roles in growth and development in rice. The tissue expression pattern of the Os GNOM1 gene was analyzed using $2300 \mathrm{bp}$ of its promoter region and $215 \mathrm{bp}$ of its coding region fused to the GUS-coding region. GUS staining of the transgenic plants revealed that $O S G N O M 1$ is expressed in the vascular tissues of roots (Figure 6B), LRs (Figure 6B), root tips (Figure 6C), anthers and lemma veins (Figure 6D and $6 \mathrm{E}$ ), and leaves (Figure 6F). Cross-sections of the stem base showed that OsGNOM1 was also expressed in the AR primordia (Figure $6 \mathrm{G}$ ). To determine whether phytohormones affected the expression of OsGNOM1, semi-quantitative RT-PCR was performed. As shown in Figure $6 \mathrm{H}$, the expression of $O s G N O M 1$ was not induced by NAA, NPA or 6-benzylaminopurine (6-BA), but it was strongly induced by abscisic acid (ABA) in both WT plants and gnom 1 mutants.

\section{Sensitivity of OsGNOM1 to BFA}

Large ARF-GEFs in endosomes can be classified into two groups, according to their response sensitivity to the fungal toxin BFA, which specifically blocks vesicle trafficking. Arabidopsis GNOM1 is sensitive to BFA [18, 21]. OsGNOM1 is a closer homolog to the Arabidopsis GNOM than to the six other rice GNOM proteins except Os02g22090 (Figure 7C). Four critical amino acids in 
Table 2 Effect of BFA on seedling growth

\begin{tabular}{lllll}
\hline Concentration of BFA $(\mu \mathrm{M})$ & SL $(\mathrm{cm})$ & SRL $(\mathrm{cm})$ & ARN & \multicolumn{2}{l}{ LRN } \\
\hline 0 & $9.26 \pm 0.75$ & $8.82 \pm 0.38$ & $4.80 \pm 0.63$ & $112.50 \pm 32.76$ \\
0.1 & $8.43 \pm 0.31$ & $7.59 \pm 0.82$ & $5.1 \pm 1.28$ & $30.35 \pm 8.24^{*}$ \\
1 & $7.35 \pm 0.40^{*}$ & $4.97 \pm 0.67^{*}$ & $4.5 \pm 1.87$ & $0 \pm 0 *$ \\
5 & $2.48 \pm 0.33^{*}$ & $1.37 \pm 0.24^{*}$ & $5.3 \pm 0.46$ & 0 \\
\hline
\end{tabular}

${ }^{1}$ BFA, brefeldin A; SL, shoot length; SRL, seminal root length; ARN, adventitious root number; LRN, lateral root number.

${ }^{2} *$ Mean significant difference $(P<0.05, t$-test).

${ }^{3}$ Measurements were obtained from 7-day-old seedlings of the Zhonghua 11 cultivar. Five plants per line were calculated, and data represented as means \pm SD.

the SEC7 domain that determine the sensitivity to BFA [18] are identical between Arabidopsis GNOM and OsGNOM1 (Figure 7A), suggesting that OsGNOM1 might be sensitive to BFA. To determine the effects of BFA on the development of AR and LR, different concentrations of BFA were applied to WT rice seedlings. While the BFA-treated rice seedlings had similar numbers of AR in comparison to control seedlings, the number of LRs decreased with BFA treatments of increasing concentrations (Figure 7B and Table 2). Thus, although BFA inhibited the initiation of LR, BFA application did not phenocopy the effect of OsGNOM1 mutation on AR development.

\section{Discussion}

Here, we report that mutants carrying four different mutations in the rice OsGNOM1 gene display defective AR initiation. OsGNOM1 proved to be highly homologous to the GNOM protein in Arabidopsis. Mutations in Arabidopsis GNOM resulted in serious defects in both embryonic and post-embryonic development [34].

Auxin is a major growth-promoting hormone for the initiation of LR primordia in Arabidopsis [3]. A previous study revealed that GNOM controlled PAT through polar localization of PIN1 [17], a component of auxin efflux carriers. In addition, some ARF-regulating factors in rice are also related to PAT, such as the ARF-activating protein involved in the localization of AUX1, an auxin influx carrier [35]. All mutant phenotypes observed in gnom1-1 to gnom 1-4, including a lack of AR, reduced number of LR, partial agravitropic response and hypersensitivity to NPA, could be explained by disrupted PAT. OsGNOM1 was expressed in a pattern parallel to that of auxin distribution (Figure 6), supporting the hypothesis that OsGNOM1 is involved in PAT. The expression of OsPINs was altered in the mutants (Figure 5), including OsPIN2, OsPIN5b and OsPIN9 (Figure 5A and 5B), which may also have contributed to the observed phenotype. Moreover, OsPIN1b, OsPIN5b and OsPIN9 are all expressed in the stem base where AR primordia form
(Wang et al., unpublished), suggesting that these PIN genes are likely involved in the formation of ARs. Since OSPIN5b and OSPIN9 have no corresponding homologous genes in Arabidopsis, the function of these genes might explain the difference between the tap root system of Arabidopsis and the fibrous root system of rice. These OsPINs function in PAT, either directly controlled by OsGNOM1 or in a feedback loop with auxin $[32,36]$. Taken together, our results suggest that OsGNOM1 is required for the initiation of AR primordia, which is mediated by OsPIN family genes.

Due to the one (gnom 1-1) or two (1-3 and 1-4) nucleotide, deletion in the GNOM1 protein-coding sequence, gnom 1-1 to 1-4 mutants cannot produce functional GNOM1 protein. Some strong allelic mutants of Arabidopsis gnom showed defects in embryonic development, mainly involving nonsense mutations before or in the central portion of the SEC7 domain [19]. Whereas rice mutants gnom 1-1 to gnom 1-4did not show any defects in embryonic development, some mutations (gnom 1-4) occurred prior to the SEC7 domain. In this study, we have mainly focused on screening for mutants with defects in root development. The more severe mutations related to embryonic development may have been missed. It is also possible that OsGNOM1 has little role in the embryonic development in rice, due to the differences in embryonic structure between dicotyledonous plants and cereals.

To date, several mutants affecting root development have been characterized in cereals [37]. Unlike arll, which only affects AR development, mutations in rice gnom 1 not only affect AR development, but also reduce the numbers of LR, suggesting that OsGNOM1 might be involved in the common mechanisms regulating the development of root components.

The fungal toxin BFA was shown to specifically inhibit the membrane trafficking of GNOM in Arabidopsis [18]. Thus, application of BFA to the WT Arabidopsis plants phenocopied the gnom mutants. Interestingly, the function of rice GNOM1 was shown to be partially resistant to BFA, despite predictions that it would be sensitive 
due to the presence of conserved amino acids (Figure 7A). An opposite pattern was observed for tobacco NtGNL1, sensitive to BFA despite predictions that it would be resistant [38]. BFA application reduced the number of LR (Figure 7B), while it did not affect the number of AR. The discrepancy in BFA sensitivity between rice and Arabidopsis might be attributable to differential GNOM protein function in the mono- and dicotyledonous plant species.

Mutation of OsGNOM1 resulted in a reduced rate of callus induction and changed the characteristics of callus in indica, but not in japonica. It is likely that OsGNOM1 may not function in precisely the same way in indica and japonica rice subspecies.

Identification of the function of the OsGNOM1 gene in root development demonstrates that some members of the SEC7 domain gene family may play an important role in organ development in plants. In total, eight SEC7 domain genes with various similarities were identified throughout the rice genome (Figure 7C). Further studies to clarify the function of these SEC7 genes utilizing transgenic approaches will greatly enhance our knowledge of the molecular mechanisms underlying rice organ development. Precise monitoring of the expression level and the tissue specificity of OsGNOM1 and its homologs may hold potential for the improvement of crop root architecture, with the ultimate goal of increasing nutrient and water uptake efficiency.

\section{Materials and Methods}

\section{Isolation of the mutants}

Rice mutant libraries of japonica Zhonghua 11 and indica variety Kasalath were constructed utilizing tissue culture techniques, EMS or ${ }^{60} \mathrm{Co}$ irradiation as described in Table 1. Four mutants (denoted as gnom1-1 to gnom 1-4) lacking ARs were identified. gnom 1-1 was from Zhonghua 11, and the gnom1-2 to gnom1-4 mutants were generated from Kasalath.

\section{Growth conditions and hormone treatments}

Seedlings of WT rice and heterozygous mutants were planted hydroponically at $\mathrm{pH}$ 5.0. The hydroponic culture solution contained $1.425 \mathrm{mM} \mathrm{NH}_{4} \mathrm{NO}_{3}, 0.323 \mathrm{mM} \mathrm{NaH}_{2} \mathrm{PO}_{4}, 0.513 \mathrm{mM}$ $\mathrm{K}_{2} \mathrm{SO}_{4}, 0.998 \mathrm{mM} \mathrm{CaCl}_{2}, 1.643 \mathrm{mM} \mathrm{MgSO}, 0.009 \mathrm{mM} \mathrm{MnCl}$, $0.075 \mathrm{mM}\left(\mathrm{NH}_{4}\right)_{6} \mathrm{Mo}_{7} \mathrm{O}_{24}, 0.019 \mathrm{mM} \mathrm{H}_{3} \mathrm{BO}_{3}, 0.155 \mathrm{mM} \mathrm{CuSO}_{4}$, $0.036 \mathrm{mM} \mathrm{FeCl}_{3}, 0.070 \mathrm{mM}$ citric acid and $0.152 \mathrm{mM} \mathrm{ZnSO}_{4}$ [13]. Rice plants were grown in growth chambers with a $12 \mathrm{~h}$ photoperiodic condition $\left(200 \mu \mathrm{mol}\right.$ photons $\left.\mathrm{m}^{-2} \mathrm{~s}^{-1}\right)$ and a temperature of $30^{\circ} \mathrm{C}$ after germination. Humidity was controlled at approximately $60 \%$. To analyze the effects of NPA on the phenotypes, $0.5 \mu \mathrm{M}$ or $1 \mu \mathrm{M}$ NPA was applied exogenously. Measurements of LR density were taken with WinRHIZO version 3.9 (Régent Instrument Inc., Québec, Canada). The gravitropic response of the primary root was determined using 20 seedlings grown for 3, 6 and 9 days. BFA dissolved in DMSO was added to the hydroponic solution with a serially diluted concentration gradient.

\section{Mapping and cloning of OSGNOM1}

A mapping $\mathrm{F}_{2}$ population was generated from the crosses between Kasalath and plants heterozygous for the gnom 1-1 mutant. OsGNOM1 was primarily mapped with SSR and STS markers, which were developed from the sequence difference japonica Nipponbare contig sequences and indica variety 93-11 sequences using $120 \mathrm{~F}_{2}$ homozygous mutant plants. The mutation was further mapped within a 90-kb region flanking an STS19 marker at a BAC clone (OSJNBa0056E06) on chromosome 3 using $962 \mathrm{~F}_{2}$ mutant plants. The DNA sequences of candidate genes were amplified from both the gnom 1-1 mutant and WT genomic DNA using three pairs of gene-specific primers (Supplementary information, Table $\mathrm{S} 2$ ), and then cloned into T-vector for sequencing. To confirm the mutation, two dCAPS markers, $\mathrm{C} 1$ and $\mathrm{C} 2$, were developed according to the sequence described previously [39] at http://helix. wustl.edu/dcaps/dcaps.html. The mutation of gnom 1-3 was confirmed by $\mathrm{Cel}$ I according to the instructions [40].

A BLAST search was performed to identify the homologs of OsGNOM1. The predicted protein sequences from these homologs were clustered using ClustalW (version 1.74, Thompson, Higgins, Gibson).

\section{Callus survey of the mutants}

To calculate the rate of callus induction, calli originating from the indica and japonica backgrounds were induced according to the protocol [41]. A total of 100 seeds from the gnom1-1 and gnom 1-2 heterozygous populations were divided into three culture dishes, and the experiment was repeated three times. After callus formation, we counted the seeds capable of being induced. The callus was then photographed after subculture. The $\mathrm{C} 1$ and $\mathrm{C} 2$ dCAPS markers were used to distinguish the homozygous mutant calli from the heterozygous population.

\section{Construction of vectors and plant transformation}

For complementation of rice gnom 1-1 mutants, a 4.8-kb cDNA fragment of wild type containing the OsGNOM1 ORF was cloned into a binary vector, modified pCAMBIA 1300 driven by the $35 \mathrm{~S}$ promoter. The homozygous embryonic calli induced from heterozygous population seeds were identified using dCAPS marker $\mathrm{C} 1$ to serve as complementation test recipients. Recipient calli were transformed with the above construct using the Agrobacterium tumefaciens (strain EHA105)-mediated transformation system as described [41]. Transgenic plants were selected on medium containing $50 \mathrm{mg} / \mathrm{l}$ hygromycin.

For the OsGNOM1 promoter::GUS construct, a 2.3-kb DNA segment upstream of OSGNOM1 and $215 \mathrm{bp}$ of the coding region was amplified by PCR and inserted into the $5^{\prime}$ end of the GUS reporter gene in $\mathrm{pBI} 101$ [9]. The resulting binary vector was transferred into calli derived from WT Zhonghua 11. All the GUSrelated transgenic calli were selected on media containing $200 \mathrm{mg} / \mathrm{l}$ geneticin.

\section{Histochemical analysis and GUS assay}

Histochemical GUS analysis and methylene blue staining of seminal roots were performed as described [33, 42]. After being stained with X-gluc buffer overnight at $37{ }^{\circ} \mathrm{C}$, shoot bases from 5-day-old seedlings were fixed in FAA for $24 \mathrm{~h}$ at $4{ }^{\circ} \mathrm{C}$ and then 
dehydrated in a graded ethanol series. Dehydrated samples were embedded in paraffin, and then sectioned into 8 - $\mu$ m-thick sections by using a rotary microtome (Microm HM325, Walldorf, Germany).

\section{RT-PCR analysis}

Total RNA was extracted from stem bases, roots, shoots and flowers of 7-day-old WT and gnom 1-1 mutant seedlings, using the TRIzol method. Some seedlings were treated by $1 \mu \mathrm{M} \alpha$-NAA, $10 \mu \mathrm{M} 6-\mathrm{BA}, 10 \mu \mathrm{M}$ NPA or $100 \mu \mathrm{M}$ ABA for $4 \mathrm{~h}$ to harvest the stem-base samples. Five micrograms of RNA samples were reversely transcribed using M-MLV reverse transcriptase (Promega, CA, USA) and an oligo(dT)18, according to the manufacturer's protocol. RT-PCR was performed using gene-specific primers (Supplementary information, Table S2). Amplification of actin cDNA was performed as a control. The PCR conditions were $94{ }^{\circ} \mathrm{C}$ for $5 \mathrm{~min}$ followed by 32 cycles of $94{ }^{\circ} \mathrm{C}$ for $30 \mathrm{~s}, 58{ }^{\circ} \mathrm{C}$ for 30 $\mathrm{s}, 72{ }^{\circ} \mathrm{C}$ for $30 \mathrm{~s}$, and then a final extension period at $72{ }^{\circ} \mathrm{C}$ for 5 min. PCR products were analyzed on $1.2 \%$ agarose gels. RTPCR analysis for the complementation test was performed using $15 \%$ PAGE gels to separate PCR products from the wild type and the mutant. Real-time qRT-PCR was performed using the SYBR Premix Ex Taq ${ }^{\mathrm{TM}}$ (Perfect Real Time) Kit (TaKaRa Biomedicals, Tokyo, Japan) on a LightCycler480 machine (Roche Diagnostics, Basel, Switzerland), according to the manufacturer's instructions.

\section{Acknowledgments}

This work was supported by the Key Basic Research Special Foundation of China (2005CB20900), the National High Technology Research and Development Program (2007AA021403, 2006AA10Z175), the National Natural Science Foundation of China (30471118 and 30770191) and the Specialized Research Fund for the Doctoral Program of Higher Education (20070335081).

\section{References}

1 Kawata S, Yamazaki K, Ishihara K, Shibayama H, Lai KL. Studies on root system formation in rice plants in a paddy. Proc Crop Sci Soc Jpn 1963; 32:163.

2 Scheres B, Benfey PN, Dolan, L. Root development. In: Somerville C, Meyerowitz E, eds. The Arabidopsis Book. American Society of Plant Biologists, Rockville, MD 2002:1-19.

3 Casimiro L, Beeckman T, Graham N, et al. Dissecting Arabidopsis lateral root development. Trends Plant Sci 2003; 8:165-171.

4 Fukaki H, Tasaka M. Hormone interactions during lateral root formation. Plant Mol Biol 2009; 69: 437-449.

5 Lucas M, Godin C, Jay-Allemand C, Laplaze L. Auxin fluxes in the root apex co-regulate gravitropism and lateral root initiation. J Exp Bot 2008; 59:55-66.

6 Hetz W, Hochholdinger F, Schwall M, Feix, G. Isolation and characterization of rtcs, a mutant deficient in the formation of nodal roots. Plant $J$ 1996; 10:845-857.

7 Inukai Y, Miwa M, Nagato Y, Kitano H, Yamauchi, A. Characterization of rice mutants deficient in the formation of crown roots. Breed Sci 2001; 51:123-129.

8 Inukai Y, Sakamoto T, Ueguchi-Tanaka M, et al. Crown rootless1, which is essential for crown root formation in rice, is a target of an auxin response factor in auxin signaling. Plant Cell 2005; 17:1387-1396.

9 Liu H, Wang S, Yu X, et al. ARL1, a LOB-domain protein required for adventitious root formation in rice. Plant $J$ 2005; 43:47-56.

10 Teale WD, Paponov IA, Palme K. Auxin in action: signalling, transport and the control of plant growth and development. Nat Rev Mol Cell Biol 2006; 7:847-859.

11 Fukaki H, Nakao Y, Okushima Y, Theologis A, Tasaka M. Tissue-specific expression of stabilized SOLITARY-ROOT/ IAA14 alters lateral root development in Arabidopsis. Plant J 2005; 44:382-395.

12 Liu PP, Montgomery TA, Fahlgren N, et al. Repression of AUXIN RESPONSE FACTOR10 by microRNA160 is critical for seed germination and post-germination stages. Plant $J$ 2007; 52:133-146.

$13 \mathrm{Xu} \mathrm{M}$, Zhu L, Shou H, Wu P. A PIN1 family gene, OsPIN1, involved in auxin-dependent adventitious root emergence and tillering in rice. Plant Cell Physiol 2005; 46:1674-1681.

14 Taramino G, Sauer M, Stauffer JL, et al. The maize (Zea mays L.) RTCS gene encodes a LOB domain protein that is a key regulator of embryonic seminal and post-embryonic shootborne root initiation. Plant J 2007; 50:649-659.

15 Shevell DE, Leu WM, Gillmor CS, et al. EMB30 is essential for normal cell division, cell expansion, and cell adhesion in Arabidopsis and encodes a protein that has similarity to Sec7. Cell 1994; 77:1051-1062.

16 Busch M, Mayer U, Jurgens G. Molecular analysis of the Arabidopsis pattern-formation gene GNOM: gene structure and intragenic complementation. Mol Gen Genet 1996; 250:681691.

17 Steinmann T, Geldner N, Grebe M, et al. Coordinated polar localization of auxin efflux carrier PIN1 by GNOM ARF GEF. Science 1999; 286:316-318.

18 Geldner N, Anders N, Wolters H, et al. The Arabidopsis GNOM ARF-GEF mediates endosomal recycling, auxin transport, and auxin-dependent plant growth. Cell 2003; 112:219230 .

19 Geldner N, Richter S, Vieten A, et al. Partial loss-of-function alleles reveal a role for GNOM in auxin transport-related, post-embryonic development of Arabidopsis. Development 2004; 131:389-400.

20 Koizumi K, Sugiyama M, Fukuda H. A series of novel mutants of Arabidopsis thaliana that are defective in the formation of continuous vascular network: calling the auxin signal flow canalization hypothesis into question. Development 2000; 127: 3197-3204.

21 Anders N, Jurgens G. Large ARF guanine nucleotide exchange factors in membrane trafficking. Cell Mol Life Sci 2008; 21:3433-3445.

22 Mayer U, Torres Ruiz RA, Berleth T, Misera S, Jurgens G. Mutations affecting body organization in the Arabidopsis embryo. Nature 1991; 353:402-407.

23 Shevell DE, Kunkel T, Chua NH. Cell wall alterations in the Arabidopsis emb30 mutant. Plant Cell 2000; 12:2047-2060.

24 Grebe M, Gadea J, Steinmann T, et al. A conserved domain of the Arabidopsis GNOM protein mediates subunit interaction and cyclophilin 5 binding. Plant Cell 2000; 12:343-356.

25 Mouratou B, Biou V, Joubert A, et al. The domain architecture 
of large guanine nucleotide exchange factors for the small GTP-binding protein Arf. BMC Genomics 2005; 6:20.

26 Anders N, Nielsen M, Keicher J, et al. Membrane association of the Arabidopsis ARF exchange factor GNOM involves interaction of conserved domains. Plant Cell 2008; 20:142-151.

27 Richter S, Geldner N, Schrader J, et al. Functional diversification of closely related ARF-GEFs in protein secretion and recycling. Nature 2007; 448:488-492.

28 Teh OK, Moore I. An ARF-GEF acting at the Golgi and in selective endocytosis in polarized plant cells. Nature 2007; 448: 493-496.

29 Muday G.K, Peer WA, Murphy AS. Vesicular cycling mechanisms that control auxin transport polarity. Trends Plant Sci 2003; 8:301-304.

30 Geldner N, Friml J, Stierhof Y, Jurgens G, Palme K. Auxin transport inhibitors block PIN1 cycling and vesicle trafficking. Nature 2001; 413: 425-428.

31 Friml J, Vieten A, Sauer M, et al. Efflux dependent auxin gradients establish the apical-basal axis of Arabidopsis. Nature 2003; 426:147-153.

32 Blilou I, Xu J, Wildwater M, et al. The PIN auxin efflux facilitator network controls growth and patterning in Arabidopsis roots. Nature 2005; 433:39-44.

33 Wang H, Taketa S, Miyao A, Hirochika H, Ichii M. Isolation of a novel lateral-rootless mutant in rice (O. sativa L.) with reduced sensitivity to auxin. Plant Sci 2006; 170:70-77.

34 Mayer U, Buttner G, Jurgens G. Apical-basal pattern forma- tion in the Arabidopsis embryo: studies on the role of the gnom gene. Development 1993; 117:149-162.

35 Zhuang X, Jiang J, Li J, et al. Over-expression of OsAGAP, an ARF-GAP, interferes with auxin influx, vesicle trafficking and root development. Plant J. 2006; 48:581-591.

36 Paponov IA, Teale WD, Trebar M, Blilou I, Palme K. The PIN auxin efflux facilitators: evolutionary and functional perspectives. Trends Plant Sci 2005; 10:170-177.

37 Hochholdinger F, Park WJ, Sauer M, Woll K. From weeds to crops: genetic analysis of root development in cereals. Trends Plant Sci 2004; 1:42-48.

38 Wang L, Liao FL, Zhu L, Peng XB, Sun MX. NtGNL1 is involved in embryonic cell division patterning, root elongation, and pollen tube growth in tobacco. New Phytol 2008; 179:81-93.

39 Neff MM, Neff JD, Chory J, Pepper AE. dCAPS, a simple technique for the genetic analysis of single nucleotide polymorphisms: experimental applications in Arabidopsis thaliana genetics. Plant J 1998; 14:387-392.

40 Till BJ, Burtner C, Comai L, Henikoff S. Mismatch cleavage by single-strand specific nucleases. Nucleic Acids Res 2004; 32:2632-2641.

41 Chen SY, Jin WZ, Wang MY, et al. Distribution and characterization of over 1000 T-DNA tags in rice genome. Plant $J$ 2003; 36:105-113.

42 Jefferson RA, Kavanagh TA, Bevan MW. GUS fusions: $\beta$-glucuronidase as a sensitive and versatile gene fusion marker in higher plants. EMBOJ 1987; 6:3901-3907.

(Supplementary information is linked to the online version of the paper on the Cell Research website.) 